\title{
Substorm classification with the WINDMI model
}

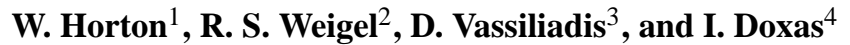 \\ ${ }^{1}$ The University of Texas at Austin, Austin, TX 78712, USA \\ ${ }^{2} \mathrm{NRC}$ at NASA/GSFC, Greenbelt, MD 20771, USA \\ ${ }^{3}$ USRA at NASA/GSFC, Greenbelt, MD 20771, USA \\ ${ }^{4}$ Department of Physics, University of Colorado, Boulder, CO 80309, USA
}

Received: 20 August 2002 - Revised: 13 September 2002 - Accepted: 5 December 2002

\begin{abstract}
The results of a genetic algorithm optimization of the WINDMI model using the Blanchard-McPherron substorm data set is presented. A key result from the large-scale computations used to search for convergence in the predictions over the database is the finding that there are three distinct types of $v_{x} B_{S}-A L$ waveforms characterizing substorms. Type I and III substorms are given by the internally-triggered WINDMI model. The analysis reveals an additional type of event, called a type II substorm, that requires an external trigger as in the northward turning of the IMF model of Lyons (1995). We show that incorporating an external trigger, initiated by a fast northward turning of the IMF, into WINDMI, a low-dimensional model of substorms, yields improved predictions of substorm evolution in terms of the $A L$ index. Intrinsic database uncertainties in the timing between the ground-based $A L$ electrojet signal and the arrival time at the magnetopause of the IMF data measured by spacecraft in the solar wind prevent a sharp division between type I and II events. However, within these timing limitations we find that the fraction of events is roughly $40 \%$ type I, $40 \%$ type II, and $20 \%$ type III.
\end{abstract}

\section{Introduction}

The need for a substorm model that is a nonlinear function of the solar-wind driver has become evident from previous numerical experiments which used the rectified solar-wind electric field, $v_{x} B_{s}$, to determine the evolution of substorm electrojet currents, as measured by the $A L$ index. The substorm database generated and analyzed by Bargatze et al. (1985) showed that at mid-ranges of substorm activity, the impulse response function had a bimodal shape with peaks at approximately 20 and $60 \mathrm{~min}$. Explicitly bimodal filters were studied by Blanchard and McPherron (1993) and Smith and Horton (1998) who found that approximately $70 \%$ of the variation in $A L$ may be explained by a bimodal low-pass fil-

Correspondence to: W. Horton (horton@physics.utexas.edu) ter. At high levels of activity, the impulse response function is unimodal and is well described by the response of a second-order (LRC) filter (Vassiliadis and Klimas, 1995). Other works used a nonlinear filter based input-output relationship, e.g. Vassiliadis et al. $(1995,1996)$, to show that improvements in a linear filter model can be made if the coefficients are dependent on both $A L$ and $v_{x} B_{S}$; Rostoker and Pascal (1990) showed that the bimodal shape of the impulse response function can also be generated by allowing the parameters of a LRC model to be time dependent.

In this work, we describe the inclusion of a substorm trigger that is initiated by a northward turning of the IMF into WINDMI, a low-dimensional substorm model (Horton and Doxas, 1996, 1998). The motivation for including a northward turning trigger is the observation that a large percentage of substorms follow the predictions of the internally-triggered low-dimensional nonlinear model WINDMI (which models the proposed type I and III events Weigel, 2000), but for a significant number of intervals it is found that this reference WINDMI model cannot provide an explanation for certain waveforms in the data; many of these waveforms have the common feature of the expansion phase occurring at a time different from what the model predicts, namely after the solar-wind driving electric field has shut down. The first efforts to eliminate this problem focused on including more physical processes in the model. Weigel (2000) introduced modifications to the reference model to include (1) the addition of a nonlinear Pedersen conductance model and (2) a region 2 to region 1 current coupling following the Siscoe (1982) model. While these extensions were able to modestly improve the model performance over a substorm database, the basic timing problem on the same subset of events remained. Turning then to look at the mathematical structure of the system, we identified that the causality inherent in the internally-triggered substorm model does not allow the expansion phase to occur after a drop in the driving convection electric field. Thus, the causality contained in the physics-based model suggests that events exist that re- 
quire an external trigger which is initiated by a drop in the solar-wind convection electric field.

We are using a broad definition of a substorm event to describe the $A L$ waveform after the IMF has been southward for a long enough time to cause a rapid increase in westward electrojet currents. We are interested in understanding the waveforms using a plasma physical model that only requires the input of the cross-tail electric field $\left(v_{x} B_{s}\right)$ generated by the convecting solar wind. Because this is a global description of substorms, high spatial resolution is not included. This limits the absolute accuracy of the model, but all of the essential physics is included. Many of the random errors are absorbed into the model parameters, which are allowed to vary within a small range. Some of the error can be attributed to incomplete magnetometer coverage in the midnight sector; this will give an error in the conversion factor from the model current to $A L$. The inclusion of other effects, such as inductive ground coupling, will increase the order of the equations, but as was found when a region 2 current loop is added, there is little difference in model performance when additional linear current loops are added. This agrees with the assumption that although the magnetosphere-ionosphere (MI) dynamics has many degrees of freedom, nearly all of the observed global behavior can be reduced to a low-dimensional set of equations.

By separating the substorm response into types, we are attempting to show how a low-dimensional physics-based model can reproduce the major observed waveforms in many isolated substorm intervals as given by the $A L$ time series. These major observed behaviors include (Weigel, 2000): (I) a bimodal response consistent with an internal trigger that is based on the near-Earth neutral line model, (II) a rapid unloading initiated by a northward IMF turning, and (III) a linear filter aspect.

Instead of ordering the intervals by activity level, we propose that the essential nonlinearity can be uncovered by separating the substorm intervals by the trigger type. This classification is a new approach to studying a substorm database and is based on frequently observed characteristics of the $v_{x} B_{s}-A L$ signature during substorms, and it provides more insight into the key input-output substorm features than the usual classification according to activity level.

Here our purpose is to show that (1) incorporating a trigger mechanism that is initiated by a northward turning of the IMF into the reference WINDMI system gives a model that can reproduce the response of substorms which have expansion phases which are inconsistent with the (internally triggered) reference model, and (2) for a large percentage of substorms, the $A L-v_{x} B_{s}$ signature clearly falls into one of the three event categories defined in Sect. 5.

\section{Model description}

We start with the reference model of WINDMI as developed in Horton and Doxas $(1996,1998)$ and Horton et al. (1998), which conserves charge and energy and describes the causal energy transfer processes between the solar wind, magnetosphere, and ionosphere during substorms. The mathematical expression of the model is given in Eqs. (1)-(6); an energy flow diagram of the multiple energy pathways is given in Horton et al. (1999). The model describes six distinct (and comparable in magnitude) energy components with six pairs of energy transfer terms. The conservation laws for the coupling terms are fundamental properties of the mathematical physics most clearly expressed through algebraic topology (Lefschetz and Solomon, 1975; Bamberg and Sternberg, 1990). The driven-damped nonlinear system of six ordinary differential equations (ODEs) is

$$
\begin{aligned}
L \frac{d I}{d t} & =V_{\mathrm{sw}}-V+M \frac{d I_{1}}{d t} \\
C \frac{d V}{d t} & =I-I_{1}-I_{p s}-\Sigma V \\
\frac{3}{2} \frac{d P}{d t} & =\Sigma \frac{V^{2}}{\Omega}-u_{0} K_{\|}^{1 / 2} \Theta\left(I-I_{c}\right) P-\frac{P}{\tau_{E}} \\
\frac{d K_{\|}}{d t} & =\alpha P^{1 / 2} V-\frac{K_{\|}}{\tau_{\|}} \\
L_{1} \frac{d I_{1}}{d t} & =V-V_{1}+M \frac{d I}{d t} \\
C_{1} \frac{d V_{1}}{d t} & =I_{1}-\Sigma_{I} V_{1} .
\end{aligned}
$$

Equations (1) and (2) describe the evolution of the crosstail potential $V=E_{y} L_{y}$ and cross tail current $I$ given the solar-wind driving potential $V_{\mathrm{sw}}=\beta v_{x} B_{s} L_{y}$, where the factor $\beta$ represents the efficiency with which the solar-wind voltage is translated to a cross-tail potential drop. The system of Eqs. (1)-(6) provides an accurate representation the standard type I isolated substorm as shown in Horton et al. (1999) and Fig. 2a.

The WINDMI model goes beyond a linear circuit based description of the interaction between the geotail current $(I)$ loop and nightside region 1 current $\left(I_{1}\right)$ loop by including the physics of an internal trigger that unloads plasma pressure through parallel mass flow on newly opened magnetic field lines. The pressure switch in Eq. (3) initiates unloading due to the magnetic field bifurcation that occurs when the cross-tail current (or the current density $j_{y}(t)=I(t) / L_{x} L_{z}$ ) reaches a critical level, $I_{c}$. The dawn-to-dusk plasma current $I$ is the sum of the pressure gradient current $I_{p s}=\alpha P^{1 / 2}$ and the collisionless, viscous stress driven, current

$$
\begin{aligned}
I_{\text {vis }} & =\int j_{y} d x d z r \text { notag } \\
& =\int d x d z\left(\widehat{\boldsymbol{y}} \cdot \boldsymbol{B} \times \nabla \cdot \pi / B^{2}\right) \propto \Sigma V,
\end{aligned}
$$

which is due to plasma convection, arises from the divergence of the ion off-diagonal momentum stress tensor $\pi$ (Horton and Doxas, 1996). The closure of the $I_{1}$ current loop subtracts from these dusk-to-dawn currents flowing from in regions obtained by mapping the auroral field lines to the geotail. 
The model also includes the dynamics of magnetosphereionosphere coupling in Eqs. (5) and (6). These two equations describe the inductive coupling between the lobe current and region 1 field-aligned currents. This is in contrast to simplified models that assume that the magnetic field lines connecting the magnetosphere to the ionosphere are equipotentials so that the magnetospheric and ionospheric electric fields are proportional, with the proportionality determined by a mapping factor (Weimer, 1994).

A direct estimate of the lobe inductance and the plasma sheet capacitance taken from Horton and Doxas (1996) is given as a parameter estimation example. From a magnetic field model such as Tysganenko (1996) we can calculate the lobe area $A$ and the effective length $L_{x}$ of the support of the geotail current density $j_{y}(x)$, then

$\mathcal{L}=\mu_{o} A / L_{x}=40 H$

for $A=\pi\left(10 R_{E}\right)^{2}$ and $L_{x}=50 R_{E}$. Horton and Doxas (1998) and Horton et al. (1998) use the Tsyganenko (1997) model to compute with more precision the electrical parameters as a function of the IMF angle, but the variation is not large. Because the plasma polarization $\rho_{m} / B^{2}$ is strongly peaked near the equatorial plane, the central plasma sheet capacitance $C$ follows from the total $\boldsymbol{E} \times \boldsymbol{B}$ flow kinetic energy

$K_{\perp}=\frac{1}{2} \int \rho_{m}(x, z) \frac{E_{y}^{2}}{B^{2}} d^{3} x \equiv \frac{1}{2} C V^{2}$.

The integral is easily evaluated using $B^{2}=B_{z}^{2}+B_{x}^{\prime 2} z^{2}$, which is valid for $|z|<3 R_{E}$, and is

$C=\frac{\pi \rho_{m}(0)}{B_{z} B_{x o}} \frac{L_{x} L_{z}}{L_{y}}=12000 \mathrm{~F}$.

Here $\rho_{m}(0)$ is the mass density at the equatorial plane and $B_{x o}=L_{z} B_{x}^{\prime}$. As a result of the dependence of the plasma sheet capacitance on $B_{z}, B_{x o}$, and $\rho_{m}(0)$, all of which vary greatly during a substorm, we allow for a large range of capacitances when optimizing. The fundamental magnetotail cavity period is $T=2 \pi(\mathcal{L} C)^{1 / 2} \simeq 1$ hour (Horton and Doxas, 1998; Horton et al., 1998). The MI coupling time scale is $T_{1}=2 \pi\left(L_{1} C_{1}\right)^{1 / 2} \simeq 5 \mathrm{~min}$. The most intricate parameter calculation involves the average central plasma sheet conductance $\Sigma$ for which we refer the reader to Horton and Tajima (1991).

In Table 1 estimated values of the fourteen parameters for the reference model are listed. These estimates have been derived and calculated previously by Horton and Doxas (1996, 1998) and Horton et al. (1998).

A case-by-case examination of the phase of the $I_{1}$ current (which is proportional to the modeled activity level, $A L$ ) increase with respect to the change in the convection electric field shows that the WINDMI model with an internal trigger (triggered when the cross-tail current reaches a critical level, $I_{c}$ ) cannot produce what we have labeled a type II substorm. An example of a type II substorm is shown in Fig. 3a, where there is first a sharp drop in the convection electric
Table 1. Estimated values of the 14 magnetospheric parameters used in the reference model defined in Eqs (1)-(6).

\begin{tabular}{ll}
\hline Parameter & Est. Value \\
\hline$L(\mathrm{H})$ & 40.0 \\
$M(\mathrm{H})$ & 1.0 \\
$C(\mathrm{~F})$ & $1.2 \cdot 10^{4}$ \\
$\Sigma(\mathrm{mho})$ & 40.0 \\
$\Omega\left(\mathrm{m}^{3}\right)$ & $1.6 \cdot 10^{24}$ \\
$u_{O}\left(\mathrm{~J}^{-1 / 2} s^{-1}\right)$ & $6.0 \cdot 10^{-11}$ \\
$I_{C}(\mathrm{~A})$ & $2.2 \cdot 10^{7}$ \\
$\alpha\left(\mathrm{A} \cdot \mathrm{Pa}^{-1 / 2}\right)$ & $1.0 \cdot 10^{12}$ \\
$\tau_{\|}(\mathrm{s})$ & $1.0 \cdot 10^{3}$ \\
$\tau_{E}(\mathrm{~s})$ & $1.0 \cdot 10^{3}$ \\
$L_{1}(\mathrm{H})$ & 12.0 \\
$C_{1}(\mathrm{~F})$ & $1.0 \cdot 10^{3}$ \\
$\Sigma_{I}(\mathrm{mho})$ & 4.0 \\
$\beta$ & 0.25 \\
\hline
\end{tabular}

field $E_{y}=V / L_{y}$ and then an increase in the westward electrojet currents. The $I_{1}-V$ phase diagram for the reference model always has a time sequence, which is restricted by the Kirchhoffian structure of the WINDMI equations, that is opposite to that of such $v_{x} B_{s}-A L$ signal. Therefore, we conclude that the type II events must be triggered by an abrupt drop in the convection electric field. The drop of the convection electric field as a straightforward consequence of an abrupt northward turning of the IMF is a scenario described by Lyons (1995) and evaluated by Blanchard et al. (2000).

In this article, we describe use of the a genetic algorithm (GA) minimization technique to rule out the possibility that these anomalous events are due to large error measures (ARV, the ratio of the mean squared error to the variance of the modeled signal) for substorm intervals in which the model optimization was stuck in a local minimum. After making sufficient runs (1000 initial population vectors) with the GA method, we conclude that there are a set of events for which the addition of an external trigger to the reference model is essential.

The set of dynamical variables is $\boldsymbol{x}=\left(I, V, P, K, I_{1}, V_{1}\right)$, and the 14 parameters, taken as constant in first order approximation, are given by

$$
\begin{aligned}
\boldsymbol{P} & =\left(L, M, C, \Sigma, \Omega, u_{o}, I_{c}, \alpha, \tau_{\|}, \tau_{E}, L_{1}, C_{1}, \Sigma_{I}, \beta\right) \\
& =\left(P^{1}, P^{2}, \ldots, P^{14}\right)
\end{aligned}
$$

The optimal parameter set $\boldsymbol{P}$ is found using the GA method to minimize the ARV metric between the model $A L$ and the measured $A L$. The values of the parameters are restricted because they are based on calculations of magnetospheric quantities such as the lobe inductance, plasma sheet capacitance, and ionospheric conductance. In Table 2 we list estimated maximum and minimum values of the parameters in the reference model. 
Table 2. Estimated physical parameter value ranges. The estimates are based on calculations outlined in the text

\begin{tabular}{lll}
\hline Parameter & Max. Value & Min. Value \\
\hline$L(\mathrm{H})$ & 100 & 10 \\
$M(\mathrm{H})$ & 2.0 & 0.5 \\
$C(\mathrm{~F})$ & $5.0 \cdot 10^{4}$ & $5.0 \cdot 10^{3}$ \\
$\Sigma(\mathrm{mho})$ & 20.0 & 5.0 \\
$\Omega\left(\mathrm{m}^{3}\right)$ & $5.0 \cdot 10^{24}$ & $5.0 \cdot 10^{23}$ \\
$u_{O}\left(\mathrm{~J}^{-1 / 2} s^{-1}\right)$ & $5.0 \cdot 10^{-9}$ & $5.0 \cdot 10^{-11}$ \\
$I_{C}(\mathrm{~A})$ & $5.0 \cdot 10^{7}$ & $5.0 \cdot 10^{6}$ \\
$\alpha\left(\mathrm{A} \cdot \mathrm{Pa}^{-1 / 2}\right)$ & $5.0 \cdot 10^{12}$ & $5.0 \cdot 10^{11}$ \\
$\tau_{\|}(\mathrm{s})$ & $1.0 \cdot 10^{3}$ & $1.0 \cdot 10^{2}$ \\
$\tau_{E}(\mathrm{~s})$ & $5.0 \cdot 10^{3}$ & $5.0 \cdot 10^{2}$ \\
$L_{1}(\mathrm{H})$ & 20.0 & 5.0 \\
$C_{1}(\mathrm{~F})$ & $1.0 \cdot 10^{3}$ & $1.0 \cdot 10^{2}$ \\
$\Sigma_{I}(\mathrm{mho})$ & 15.0 & 5.0 \\
$\beta$ & 0.85 & 0.10 \\
\hline
\end{tabular}

\section{Northward turning trigger description}

Following Lyons (1995), we briefly give the argument supporting the triggering of substorms due to a northward turning of the IMF. To develop a field-aligned current in a direction consistent with substorm currents, a divergence in the pressure must form along the drift shells. If the plasma pressure $P$ is isotropic along the flux tubes, the field-aligned current mapped to the ionosphere, $j_{\| I}$, can be written as (Vasyliunas, 1970, 1984)

$j_{\| I}=\frac{B_{I}}{B_{E}} \widehat{\boldsymbol{b}} \cdot \nabla P \times \nabla \mathcal{V}$,

where the flux tube volume is $\mathcal{V}=\int d s / B$, and the gradients are evaluated in the equatorial plane. Here $B_{I}$ and $B_{E}$ are magnitudes of the magnetic field at the ionosphere and the equatorial plane, respectively, for the flux tube.

Using the particle simulation results of Peroomian and Ashour-Abdalla (1996), and a cross polar cap potential of $40 \mathrm{kV}$, Lyons (1995) finds a significant azimuthal temperature gradient in the substorm current wedge region. Assuming that this temperature gradient exists as a result of a steady dawn-to-dusk electric field that has existed for at least $30 \mathrm{~min}$, and the convection electric field is suddenly reduced to zero at $t=t_{o}$ as a result of a northward IMF turning, an expression for the time rate of change of the pressure gradient $\partial P / \partial y$ can be found. Using a magnetic field model, the transient $j_{\|}$that is driven by the azimuthal pressure gradient can be shown to generate an increase in the westward electrojet that causes a change in the ground magnetic field of several hundred nanotesla.

The above description does not provide a self-consistent theory of the triggering process because it only provides the time evolution of the pressure and wedge currents immediately after the northward turning. From the above estimates, we know that the reduction in the electric field due to a northward turning will give a rapid increase in field-aligned currents on a very short time scale, much like the type I triggering in the reference WINDMI model. To include this external trigger into WINDMI in a way similar to the type I trigger, we assume that immediately before and after a northward turning event the dynamics follow the time evolution of the reference model. In the updated (WINDMI2000) model we assume that the triggering takes place on a rapid time scale, and the northward-turning initiated current, $I_{n t}$, is zero initially. After a northward turning, we assume that $I_{n t}$ is ramped up to $\delta I$ over $10 \mathrm{~min}$ and then returns to zero. Similar to the type I triggering, when $I_{n t}$ is initiated, there will be an enhanced westward electrojet current. A more thorough analysis of different data-derived triggers based on a more complete description of the triggering mechanism will be presented in a future work.

\section{Optimization method}

We are optimizing the parameters of a nonlinear set of differential equations that have a wide variety of possible dynamics including limit cycles, chaotic attractors, and fixed points (Smith et al, 2000). Because this type of minimization, which involves optimizing the parameters of a nonlinear set of differential equations, is not a standard problem, three minimization approaches were used. The best results were obtained using the genetic algorithm approach.

The two other minimization methods considered were random grid search and gradient descent. For a random grid search, the computation time is large because the number of iterations required scales with $N_{p}^{N}$, where $N$ is the number of grid points per parameter, and $N_{p}$ is the number of parameters. Gradient descent algorithms have difficulty when encountering local minima and require many iterations for convergence. There are several modifications which can be made to speed the convergence of the basic gradient descent method, including, for example, the addition of acceleration or inertial terms or by using a conjugate gradient step. The choice of method used is dependent on the type of problem being considered. The methods based on gradient descent minimization are appropriate for error landscapes which have only one global minimum. If only few local minima exist, then the algorithm can be restarted several times with different initial conditions. Because the error landscape has many local minima, this method becomes inefficient and a global method such as the genetic algorithm, which allows for movement out of local minima, is needed.

Because of the time required for a random grid search is prohibitive and gradient descent algorithms have difficulty when encountering local minima, we turn to an optimization method that has the advantages of both, but requires less computation. The $G A$ method can handle both a search on an error function that has many local minima and a parameter set with components which are are not completely indepen- 


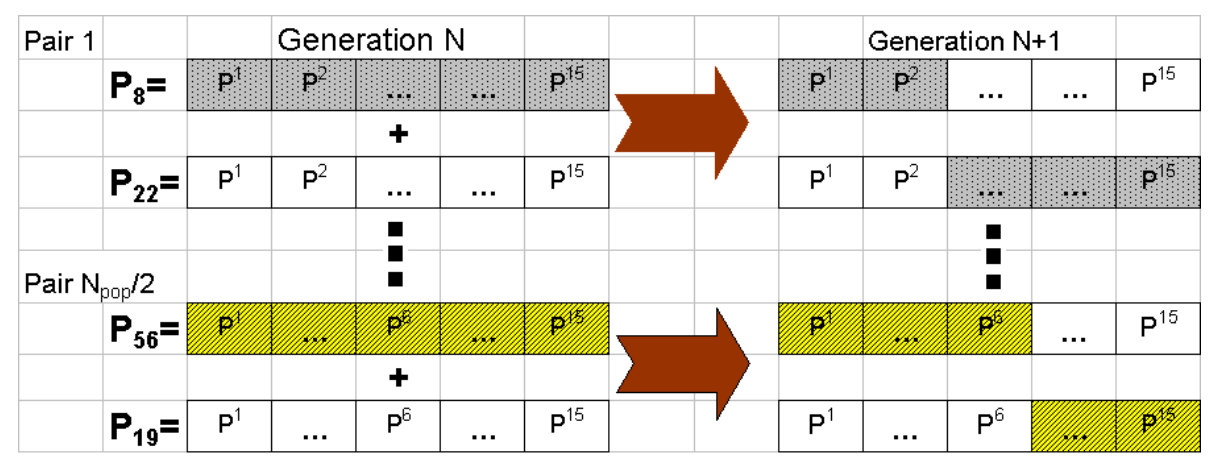

Fig. 1. Diagram of genetic algorithm mating procedure. Randomly chosen parameter vectors from an initial generation are combined to form a new generation of parameter vectors. The choice of split position $\mathcal{S}$ is denoted by a color break is chosen at random for each mating pair.

dent. The essential features for implementing the algorithm are now outlined.

The minimization procedure follows the standard genetic algorithm technique in which a population of parameter vectors $\boldsymbol{P}$ are tested, sorted according to ARV, and then split and re-combined (Vose, 1999). In this problem an initial set of 1000 parameter vectors was used.

The problem is to minimize the error function $\operatorname{ARV}=\operatorname{ARV}(\boldsymbol{P})$, where the parameter vector $\boldsymbol{P}$ has elements that are the fourteen physics parameters of WINDMI:

$$
\begin{aligned}
\boldsymbol{P} & =\left(P^{1}, P^{2}, \ldots, P^{14}\right) \\
& =\left(\mathcal{L}, \Sigma, C, \ldots, \Sigma_{I}\right)
\end{aligned}
$$

which are bounded by the maximum and minimum estimates defined in Table 2

$\mathbf{P}_{\text {max }}=\left(\mathcal{L}_{\text {max }}, \ldots, \Sigma_{I_{\text {max }}}\right)$
$\mathbf{P}_{\text {min }}=\left(\mathcal{L}_{\text {min }}, \ldots, \Sigma_{I_{\text {min }}}\right)$.

The procedure is then:

(a) Create $2 N_{\text {pop }}$ "population" vectors $\boldsymbol{P}_{j}$ whose elements are random samples in the range $P_{\min }^{i} \leq P^{i} \leq P_{\max }^{i}$

$$
\begin{gathered}
\boldsymbol{P}_{1}=\left(\mathcal{L}^{1}, \ldots, \Sigma_{I}^{1}\right) \\
\vdots \\
\boldsymbol{P}_{2 N_{\mathrm{pop}}}=\left(\mathcal{L}^{2 N_{\mathrm{pop}}}, \ldots, \Sigma_{I}^{2 N_{\mathrm{pop}}}\right)
\end{gathered}
$$

(b) Calculate the error measure $\operatorname{ARV}\left(\boldsymbol{P}_{j}\right)\left(j=1 \ldots 2 N_{\text {pop }}\right)$ and keep only half of the vectors $\left(N_{\text {pop }}\right)$ corresponding to the lowest error.

(c) Mate these two populations and create a new generation of $\boldsymbol{P}_{j}$ by (1) randomly choosing $N_{\text {pop }} / 2$ mating pairs which will give $N_{\text {pop }}$ offspring, and (2) randomly choosing a split point $\mathcal{S}$ in parameter vector $(1 \leq \mathcal{S} \leq 13)$ as diagrammed in Fig. 1.

(d) Introduce mutation (which can push the solution out of local minima) by choosing $N_{m}$ vectors from $N_{\text {pop }}$ and replacing the value of a parameter with a random value in its range.

(e) Repeat (c)-(d) until a stopping criterion is satisfied, for example

$$
\begin{aligned}
& \left.\overline{A R V}\right|^{\text {top5 }} \text { at generation } \mathrm{k} \\
& \left.\approx \overline{A R V}\right|^{\text {top5 }} \text { at generation } \mathrm{k}+1 \text {. }
\end{aligned}
$$

The stopping criterion is usually found in 4 to 5 generations. In all of the results that follow, the genetic algorithm minimization method was used to determine the optimal parameter set. We find that this method is the most reliable and efficient in minimizing WINDMI, and for this reason all reported results were generated using this scheme. An optimal parameter set is determined for each individual substorm so that the reported $A R V$ represents the in-sample prediction quality.

\section{Optimization results}

The WINDMI validation problem on type II events was thought to be due to the minimization procedure not finding the correct parameter values. After a thorough search using the genetic algorithm method, we conclude that there are type II events that are not described by the reference WINDMI model. Not every substorm interval we consider is readily identifiable as following exactly one type. From considering the events on a case-by-case basis, it is found that a significant percentage can be identified as following one of the following three types. Below we define these three categories and give representative examples of each as identified by the minimization performed on each substorm interval in the Blanchard-McPherron data set (Blanchard and McPherron, 1995).

\subsection{Type I}

In Fig. 2a, an example of the $v_{x} B_{s}-A L$ response for an isolated substorm with a type I triggering is shown. A type I 

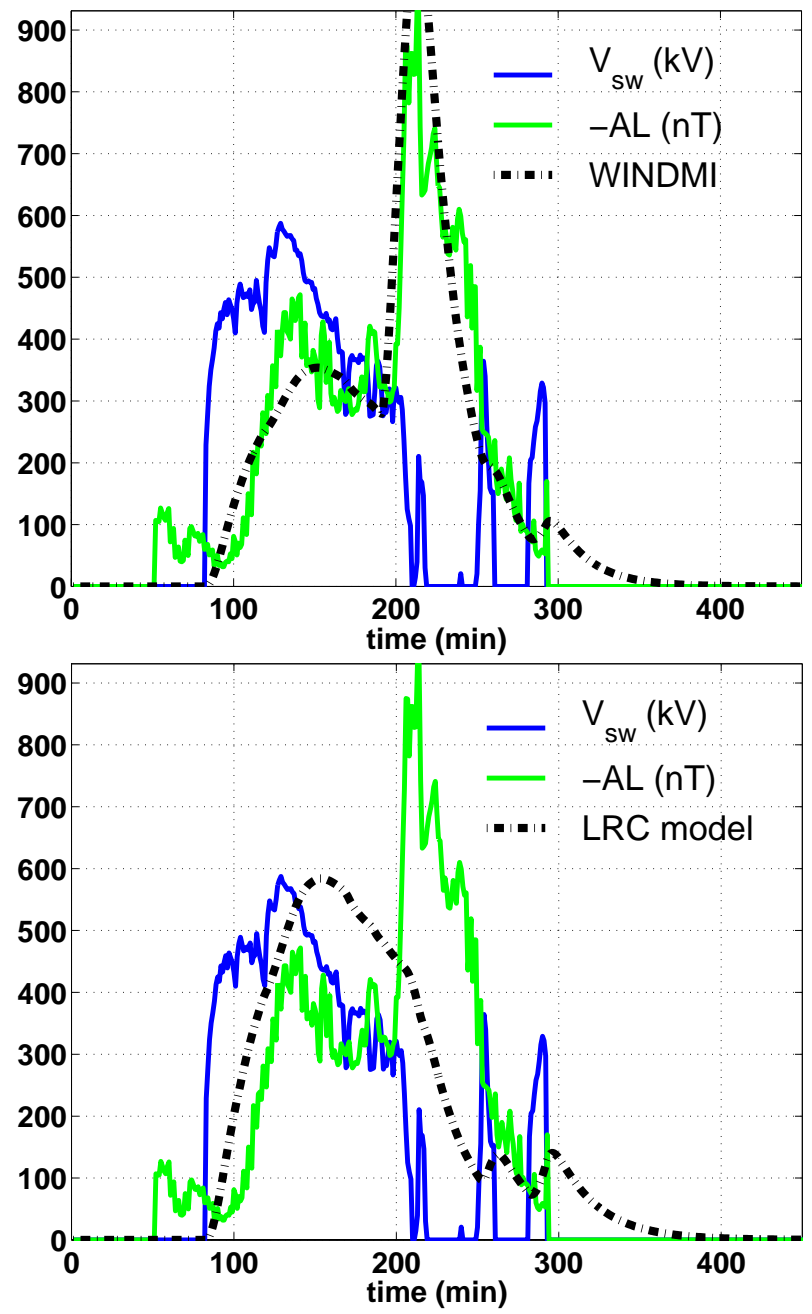

Fig. 2. A type I substorm example from the Blanchard-McPherron data set (case 35: Aug. 18, 1978). Top Panel: The optimized WINDMI system captures the rapid rise in $A L$ that begins at $t=$ $200 \mathrm{~min}$. The ARV is 0.12. Bottom panel: The optimized LRC filter prediction is shown to miss the rapid rise in $A L$ which occurs before the rapid reduction in $v_{x} B_{S}$. The ARV is 0.45 .

triggered substorm is defined to follow the response of what has previously been identified as a "canonical" substorm (Kamide and Baumjohann, 1993). After a sharp southward turning, the IMF remains steady or decreases and $A L$ grows steadily. A substorm is triggered when the energy stored in the magnetotail reaches a critical point and some of this energy is suddenly unloaded onto the ionosphere via nightside region 1 currents. The $A L$ time series shows a rapid decrease during this time. After peaking, $A L$ will return to zero in a characteristic time, provided that the IMF has turned northward or is substantially reduced. If $B_{z}$ of the IMF remains southward, as in the magnetic cloud event studied by Farrugia et al. (1993), a 50 min time between substorms is typical. Two internally triggered models (1) the Faraday Loop model (Klimas et al., 1994, 1996) and (2) the WINDMI model (Horton et al., 1998; Smith et al, 2000) reproduce
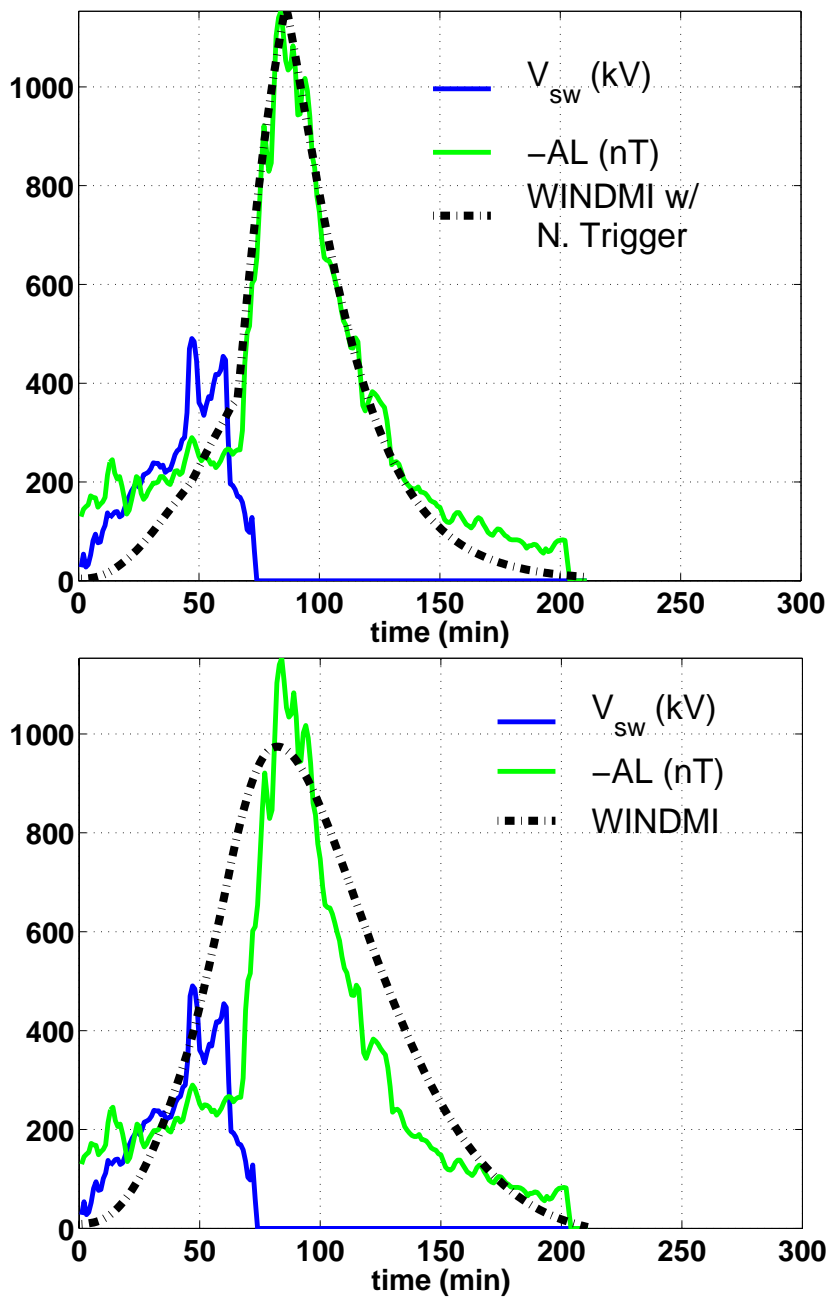

Fig. 3. Top Panel: A type II substorm example from the BlanchardMcPherron data set (case 10: 4 April 1979). The onset which occurs at $t=65$ min immediately follows a sharp reduction in $v_{x} B_{S}$. The dark thick line is $v_{x} B_{S}$, the thick light line is $A L$, and the dotted line is the response of WINDMI. The reference WINDMI response follows the general shape of $A L$, but the rapid onset phase is not evident. The ARV is 0.30 . Bottom Panel: The dark thick line is $v_{x} B_{S}$, the thick light line is $A L$, and the dotted line is the response of WINDMI with a northward turning trigger initiated at $t=65 \mathrm{~min}$. The ARV is 0.06 .

well this irregular, episodic storage-unloading behavior. The phasing of the current growth, the expansion phase, and the recovery phase are correctly given by these causal, internally triggered models. In Fig. 2a, the result of a minimization using the WINDMI model is shown. The model predicts an unloading to occur after 110 min of southward IMF (at $t=200 \mathrm{~min}$ ). Figure $2 \mathrm{~b}$ shows that the optimized response of a LRC model cannot capture the rapid unloading event.

\subsection{Type II}

In Fig. 3a, the $v_{x} B_{S}-A L$ response of WINDMI for an isolated substorm is shown. In this case the IMF turns north- 
ward before the energy stored in the magnetosphere has reached the critical level required for a type I triggering to occur. The stored energy is unloaded following the northward turning of the IMF. The triggering of a substorm by a northward turning has been examined before (Lyons, 1995; Rostoker, 1983; McPherron et al., 1986); Blanchard et al. (2000) gives a detailed analysis of the relationship between IMF conditions and substorm onsets. This work provides evidence of a causal relationship between northward IMF turnings and substorm onsets and conclude that a majority of substorms are a result of a northward turning of the IMF.

The predictions from the reference WINDMI model, shown in Fig. 3a, did not capture the rapid unloading event. In Fig. 3b, the model response is shown when the northward turning trigger mechanism is included into the updated WINDMI model. The model now captures the rapid expansion feature of the substorm. In terms of the error measure, the ARV is reduced to 0.06 for this substorm interval.

Examination of type II events shows that there is first a sharp decrease in the convection electric field $E_{y}=V / L_{y}$ followed by an increase in the westward electrojet current as measured by the $A L$ index. The $I_{1}-V$ phase diagrams for these cases have the reverse time ordering than that of an internally triggered event. Therefore, we conclude that these type II events are triggered by an abrupt drop of the convection electric field.

When the northward turning is fast, the Earthward convection of hot ion plasma stops, a strong dawn-dusk asymmetry develops, and there is an abrupt dipolarization of the near-Earth magnetic field Lyons (1995). After eliminating the weak type III substorms that are well fit by the reference WINDMI model, we find that approximately $40 \%$ of the Blanchard-McPherron database is of type II. This is a strong revision of the classical substorm picture of a nearEarth neutral line being the first event in the substorm evolution. Lyons (2000) argues that perhaps even a majority of substorm events are type II.

\subsection{Type III}

We define a type III substorm as one that is well described by a linear second order filter (LRC filter) with parameters that are within a range of estimates based on magnetospheric scales (Weimer, 1994; Horton and Doxas, 1996). The WINDMI model with no triggering can be reduced to a model with a response function near that of a LRC filter. Figure 4 shows the response of a substorm when no triggering occurs. The $A L-v_{x} B_{s}$ response is captured by the reference WINDMI model with no triggering. The main feature of a type III substorm is that all of the significant $A L$ variation is accounted for by a LRC filtering of the solar-wind driver. There may be abrupt increases in $A L$, but they are consistent with a linear second order filtering of $v_{x} B_{s}$. Bargatze et al. (1985) and Vassiliadis et al. (1996) show that as activity level increases, the impulse response function loses its second mode at $50 \mathrm{~min}$, and its shape becomes similar to that of a LRC filter. There are several proposed explanations of

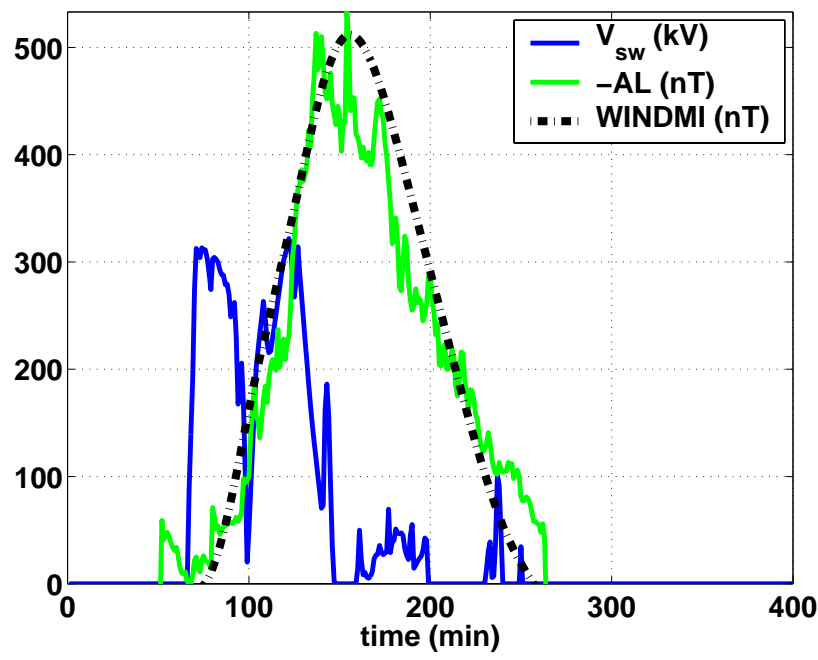

Fig. 4. Example of a type III substorm (case 98: 9 Oct. 1978). The $A L-v_{x} B_{S}$ response is predicted accurately using the WINDMI model model with no triggering.

this. For example, the second peak merges with the first, or no triggering has occurred, but for the purposes of classification we do not make this distinction. This is generally the behavior for large substorms or storms, and the predictions of a linear or near-linear filter are typically very good over a long period of time; an example of this is shown in Goertz (1993).

\section{Discussion and conclusions}

We have demonstrated that substorms can be classified into three types defined by the behavior of the WINDMI model. Because the MI system is nonlinear, to fully understand the system, one must have a complete description of the types of nonlinearities involved. This point is supported by the observation that, with a northward turning triggering mechanism, some of the second peak in impulse response filters can be attributed to northward turning events that occur after approximately 60-min of southward IMF. The classification of substorms allows for a more in-depth interpretation of correlation and impulse response studies of the relationship between solar-wind measurements and substorm features. We have provided reasons why substorm triggering needs to be taken into account when interpreting these results and shown that although part of the $A L$ signal cannot be predicted due to the stochastic nature of the solar-wind, much of its variability can be explained by the random timing of the trigger event because if the trigger time is known, the variability is significantly reduced.

The introduction of a northward turning trigger into the WINDMI model can explain the inability of both a linear filter model and the reference WINDMI model to provide a prediction of substorm electrojet currents, as measured by the $A L$ index, for a substantial fraction of isolated substorm 
events. One advantage of using the WINDMI model to describe the evolution of a substorm is that the details of the fast onset physics are separated from the longer time scale dynamics. The northward turning triggering is included explicitly in this analysis without the use of a dynamical model for its evolution, and its inclusion leads to a better model over a large isolated substorm database.

Two northward turning trigger parameters were introduced to the WINDMI model. Using a simple model for $\delta I$ in the range of 0.5MA-2.0MA and allowing $I_{n t}$ to be switched on for $10 \mathrm{~min}$, and fitting each interval with an optimized set of parameters, we find that the average ARV for the northward turning WINDMI model is 0.30 , while that of a LRC filter is 0.50 . This supports the hypothesis that, because the expansion phase physics are on a different time scale than the evolution of the dynamics contained in the low-order model, a high degree of accuracy can be obtained without their inclusion.

Using large-scale computations with several optimization methods, we have eliminated the possibility that the datamodel disagreement in what we have labeled as type II events is due to the use of unsuitable parameters in the reference model. After extensive minimization studies, we found that there was a significant set ( 40\%) of substorm events for which convergence of the predictions of the reference model and the data were not achieved. After eliminating the possibility of other physical effects changing this negative result, including a nonlinear ionospheric conductance and a region 2 coupling (Weigel, 2000), we conclude that an external, solarwind based, trigger mechanism initiated by an abrupt northward turning of the IMF is required. With the quantitative, physics-based model WINDMI, a division between the two types of substorms is firmly established.

While the Lyons (1995) northward turning description is used to motivate a model that solves this problem, we must acknowledge that there is some observational evidence against this scenario. The results of Mozer (1971) show that the westward electric field in the midnight sector increases at substorm onset; this westward electric field maps to the magnetosphere as the convection electric field. Moreover, one does not always find evidence for a decrease of earthward convection in the plasma sheet at the time of a northward turning. For these reasons, the new model needs further testing with other hypotheses for type II events.

A key point here is that by using a physics model, there is a definite causal time ordered sequence of events for the unloading events. From a histogram of ARVs, we found that approximately $40 \%$ of the events are of the classical storageunloading type and $40 \%$ are type II events. The precise division between the two types of unloading events will remain uncertain until better methods are found for timing the arrival of the solar wind at the Earth's magnetopause. Weimer (2001) has developed such a model, and it can reduce the timing-analysis uncertainty to $10 \mathrm{~min}$ or less in some cases. Moreover, because the essential, or most prominent, nonlinearity has been identified as the trigger mechanism, analysis must take this into account when attempting to describe or model substorms with filters. This suggests, in accord with Blanchard et al. (2000), that further prediction improvements will be obtained by identifying the time of triggering. Future directions of this modeling effort include coupling to a short time scale physics-based model that can more accurately predict the onset time for both types of triggers.

Acknowledgements. The work was supported by the NSF Grant No. ATM-9907637 and NASA Grant NAG5-6198. Computing resources were provided by the Texas Advanced Computing Center (TACC).

\section{References}

Baker, D. N., Pulkkinen, T. I.,Büchner, J., and Klimas, A. J.: Substorms: A global instability of the magnetosphere-ionosphere system, J. Geophys. Res., 104, 14 601-14 611, 1999.

Bamberg, P. and Sternberg, S.: A course in Mathematics for Students of Physics, Cambridge University Press, 2, 407-636, 1990.

Bargatze, L. F., Baker, D. N., McPherron R. L., and Hones, E. W.: Magnetospheric response to the IMF: substorms, J. Geophys. Res., 90, 6387, 1985.

Bishop, C. M.: Neural Networks for Pattern Recognition, Oxford University Press, 1995.

Blanchard, G. T. and McPherron, R. L.: A bimodal representation of the response function relating the solar wind electric field to the $A L$ index, Adv. Space Res., 13, 71, 1993.

Blanchard G. T. and McPherron, R. L.: Analysis of the linearresponse function relating $\mathrm{AL}$ to $\mathrm{VBs}$ for individual substorms, J. Geophys. Res., 100 (A10), 19 155-19 165, 1995.

Blanchard, G. T., Lyons, L. R., and Spann, J.: Predictions of substorms following northward turnings of the interplanetary magnetic field, J. Geophys. Res., 105, 375, 2000.

Blomberg, L. G. and Marklund, G. T.: The influence of conductivities consistent with field-aligned currents on high-latitude convection patterns, J. Geophys. Res., 93, 14 493, 1988.

Box, G. E. P. and Jenkins,G. M.: Time Series Analysis: Forecasting and Control, San Francisco Holden-Day, 1976.

Doxas, I. and Horton, W.: Magnetospheric dynamics from a lowdimensional nonlinear dynamics model, Phys. Plasmas, 6, 3, 17, 1999.

Farrugia, C. J., Freeman, M. P., Burlaga, L. F., Lepping, R. P., and Takahashi, K.: The earth's magnetosphere under continued forcing: Substorm activity during the passage of an interplanetary magnetic cloud, J. Geophys. Res., 98, 7657, 1993.

Goertz, C. K., Shan, L. H., and Smith, R. A.: Prediction of geomagnetic activity, J. Geophys. Res., 98, (A5), 7673-7684, 1993.

Goode, B., Doxas, I., Cary, J. R., and Horton, W.: Differentiating between colored random noise and deterministic chaos with the root mean squared deviation, J. Geophys. Res., 106, 21277 , 2001.

Heppner, J. P. and Maynard, N. C.: Empirical high-latitude electric field models, J. Geophys. Res., 92(A5), 4467-4489, 1987.

Horton, W. and Tajima, T.: Transport from Chaotic Orbits in the Geomagnetic Tail, Geophys. Res. Lett., 18, 1583-1586, 1991, and a recent extension by R. Numata and Z. Yoshida, Phys. Rev. Lett., 2002.

Horton, W. and Doxas, I.: A low-dimensional energy conserving state space model for substorm dynamics, J. Geophys. Res., 101, $27223,1996$. 
Horton, W. and Doxas, I.: A low-dimensional dynamical model for the solar wind driven geotail-ionosphere system, J. Geophys. Res., 103, 4561, 1998.

Horton, W., Pekker, M., and Doxas, I.: Magnetic energy storage and the nightside magnetosphere-ionosphere coupling, Geophys. Res. Lett., 25, 4083, 1998.

Horton, W., Smith, J. P., Weigel, R., Crabtree, C., Doxas, I., Goode, B., and Cary, J.: The solar-wind driven magnetosphere-ionosphere as a complex dynamical system, Phys. Plasmas, 6, 11, 1-7, 1999.

Kallio, E. I., Pulkkinen, T. I., Koskinen, H. E. J., Viljanen, A., Slavin, J. A., and Ogilvie, K.: Loading-unloading processes in the nightside ionosphere, Geophys. Res. Lett., 27, 11, 16271630,2000

Kamide, Y. and Baumjohann, W.: Magnetosphere-Ionosphere Coupling, Physics and Chemistry in Space, 23, Springer-Verlag, 1993.

Klimas, A. J., Baker, D. N., Vassiliadis, D., and Roberts, D. A.: Substorm recurrence during steady and variable solar wind driving: Evidence for a normal mode in the unloading dynamics of the magnetosphere, J. Geophys. Res. 99, A8, 14 855, 1994.

Klimas, A. J., Vassiliadis, D., Baker, D. N., and Roberts, D. A.: The organized nonlinear dynamics of the magnetosphere, J. Geophys. Res., 101, 13 089, 1996.

Klimas, A. J., Valdivia, J. A., Vassiliadis, D., Baker, D. N., Hesse, M., and Takalo, J.: Self-organized criticality in the substorm phenomenon and its relation to localized reconnection in the magnetospheric plasma sheet, J. Geophys. Res., 105, 18 765, 2000.

Lefschetz, S.: Applied Mathematical Sciences, in: Applications of Algebraic Topology: Graphs and Networks: the PicardLefschetz Theory and Feynman Integrals, Springer-Verlag, New York, Vol. 16, pp. 1884-1972, 1975.

Lui, A. T. Y., Chapman, S. C., Liou, K., Newell, P. T., Meng, C. I., Brittnacher, M., and Parks, G. K.: Is the dynamic magnetosphere an avalanching system? Geophys. Res. Lett., 27, 911, 2000.

Lyons, L. R.: A new theory for magnetospheric substorms, J. Geophys. Res., 100, $19069,1995$.

Lyons, L. R.: Geomagnetic disturbances: characteristics of, distinction between types, and relations to interplanetary conditions, J. Atmos. Sol.-Terr. Phy., 62, 1087-1114, 2000.

McPherron, R. L., Terasawa, T., and Nishida, A.: Solar Wind Triggering of Substorm Expansion Onset, J. Geomag. Geoelect., 38, 1089-1108, 1986.

Mozer, F. S.: Origin and effects of electric fields during isolated magnetospheric substorms, J. Geophys. Res., 76, 31, 7595, 1971.

Peroomian, V. and Ashour-Abdalla, M.: Population of the nearEarth magnetotail from the auroral zone, J. Geophys. Res., 101, 15,387, 1996.

Press, W. H., Teukolsky, S. A., and Flannery, B. P.: Numerical Recipes in C, Cambridge University Press, 1992.

Rostoker, G. and Pascal, F.: Dependence of the response of the magnetosphere ionosphere current systems on the preconditioning of the auroral oval and on the level of the solar terrestrial interaction, Can. J. Phys., 68, 74-80, 1990.

Rostoker, G.: Triggering of expansive phase intensifications of magnetospheric substorms by northward turnings of the interplanetary magnetic field, J. Geophys Res., 88, 6981-6993, 1983.

Robinson, R. M., Vondrak, R. R., Miller, K., Dabbs, T., and Hardy, D.: On calculating ionospheric conductances from the flux and energy of precipitating electrons, J. Geophys. Res., 92, 22652569, 1988.

Siscoe, G. L.: Energy coupling between regions 1 and 2 Birkeland current systems, J. Geophys. Res., 87, A7, 5124, 1982.

Smith, J. P. and Horton, W.: Analysis of the bimodal nature of solar wind-magnetosphere coupling, J. Geophys. Res., 103, A7 $14917,1998$.

Smith, J. P., Thiffeault, J.-L., and Horton, W.: Dynamical range of the WINDMI model: An exploration of possible magnetospheric plasma states, J. Geophys. Res., 105, A6, 12 983, 2000.

Tsyganenko, N. A.: An empirical model of the substorm current wedge, J. Geophys. Res., 102, A9, 19935-19941, 1997.

Vassiliadis, D., Klimas, A. J., Baker, D. N., and Roberts, D. A.: A description of solar wind-magnetosphere coupling based on nonlinear filters, J. Geophys. Res., 100 (A3), 3495-3512, 1995a.

Vassiliadis, D. and Klimas,A. J.: On the uniqueness of linear moving-average filters for the solar wind-auroral geomagnetic activity coupling, J. Geophys. Res., 100(A4), 5637-5641, 1995b.

Vassiliadis, D., Klimas, A. J., Baker, D. N., and Roberts, D. A.: The nonlinearity of models of the $v B$ South coupling, J. Geophys. Res., 101(A9), 19779-19,787 1996.

Vasyliunas, V. M.: Mathematical models of magnetospheric convection and its coupling to the ionosphere, in: Particles and Fields in the Magnetosphere, (Ed) McCormac, B. M., D. Reidel, (Hingham, MA, 1970) pp. 60-71. 1970.

Vasyliunas, V. M.: Fundamentals of current description, in: Magnetospheric Currents, (Ed) Potemra, T. A., American Geophysical Union, Washington, D. C. p. 63, 1984.

Vose, M. D.: The Simple Genetic Algorithm: Foundations and Theory, Cambridge Univ. Press, Cambridge, MA, 1999.

Weigel, R. S., Horton, W., Tajima, T., and Detman, T.: Forecasting auroral electrojet activity from solar wind input with neural networks, Geophys. Res. Lett. , 26, 1353, 1999.

Weigel, R. S.: Prediction and modeling of magnetospheric substorms, Ph.D. thesis, University of Texas at Austin, 2000.

Weimer, D. R.: Substorm time constants, J. Geophys. Res., 1999, A6, $11005,1994$.

Weimer, D. R., Ober, D. M., Maynard, N. C., Burke, W. J., Collier, M. R., McComas, D. J., Ness, N. F., and Smith, C. W.: Variable time delays in the propagation of the interplanetary magnetic field, J. Geophys. Res., 107(A8), doi:10.1029/2001JA009102, 2002 . 